Supporting Information

\title{
Synthesis and Pharmacological Evaluation of $\left[{ }^{11} \mathrm{C}\right] 4-$ Methoxy- $N$-[2-(thiophen-2-yl)imidazo[1,2-a]pyridin-3-yl]benzamide as a Brain Penetrant PET Ligand selective for the $\delta$-Subunit- Containing $\gamma$-Aminobutyric Acid Type A Receptors
}

Elina T. L’Estrade, $₫ \uparrow \sqcap \ddagger$ Hanne D. Hansen, $\dagger \ddagger$ Christina Falk-Petersen, $§$ Anne Haugaard,§ Nane GriemKrey, § Sascha Jung, \| Hartmut Lüddens, ${ }^{a}$ Tanja Schirmeister, $\|$ Maria Erlandsson, $\sqcap$ Tomas Ohlsson, $\sqcap$ Gitte M. Knudsen, ${ }^{\dagger}$ Matthias M. Herth,,$\$ \ddagger$ Petrine Wellendorph, ${ }^{\S}$ Bente Frølund $\S^{*}$

${ }^{\S}$ Department of Drug Design and Pharmacology, Faculty of Health and Medical Sciences, University of Copenhagen, Universitetsparken 2, 2100 Copenhagen, Denmark

+Neurobiology Research Unit and CIMBI, Copenhagen University Hospital, Rigshospitalet, Blegdamsvej 9, 2100 Copenhagen, Denmark

ח Radiation Physics, Nuclear Medicine Physics Unit, Skånes University Hospital, Barngatan 3, Lund 222 42, Sweden

${ }^{\ddagger}$ Department of Clinical Physiology, Nuclear Medicine and PET, University Hospital Copenhagen, Rigshospitalet

Blegdamsvej 9, 2100 Copenhagen, Denmark

aDepartment of Psychiatry and Psychotherapy, Falculty of Health and Medical Sciences, University of Medical Center Mainz, D-55131, Germany

\|Institute of Pharmacy \& Biochemistry, Johannes Gutenberg University Mainz, D-55128, Germany

Corresponding Author

* Phone: +45 353364 95. E-mail: bfr@sund.ku.dk

\section{Contents}

Results

Selectivity profiling:

Radiolabeling of $\left[{ }^{11} \mathrm{C}\right] \mathrm{DS} 2 \mathrm{OMe}\left(\left[{ }^{11} \mathrm{C}\right] 1\right)$...... 2

Distribution volumes and metabolism of $\left[{ }^{11} \mathrm{C}\right] 1$. .3

Radiometabolism in pigs. . .4 


\section{Results}

Selectivity profiling:

Supporting Table S1: In the table is included the targets for which DS2OMe showed less than $50 \%$ inhibition at $10 \mu \mathrm{M}$ in the National Institute of Mental Health's Psycoactive Drug Screening Program (NIMH-PDSP).

\begin{tabular}{|c|c|c|c|}
\hline \multicolumn{4}{|c|}{ Receptors (<50\% inhibition at $10 \mu \mathrm{M})$} \\
\hline 5-HT1A & Alpha1A & D4 & M2 \\
\hline 5-HT1B & Alpha1B & D5 & M4 \\
\hline 5-HT1D & Alpha1D & DAT & M5 \\
\hline 5-ht1e & Alpha2A & DOR & MOR \\
\hline 5-HT2A & Alpha2B & H1 & NET \\
\hline 5-HT2B & Beta1 & H2 & SERT \\
\hline 5-HT2C & Beta2 & H3 & Sigma 1 \\
\hline 5-HT3 & Beta3 & H4 & Sigma 2 \\
\hline 5-ht5a & D1 & KOR & \\
\hline 5-HT6 & D2 & M1 & \\
\hline 5-HT7 & D3 & M3 & \\
\hline
\end{tabular}

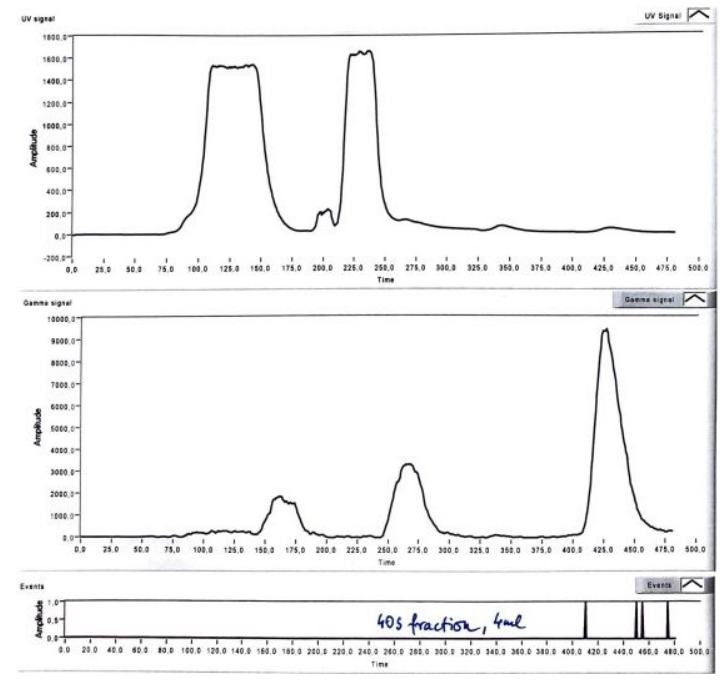

Supporting Figure S1: Semi-preparative HPLC chromatogram of radiolabeling of $\left[{ }^{11} C\right] D S 2 O M e ~\left(\left[{ }^{11} C\right] \mathbf{1}\right)$. 

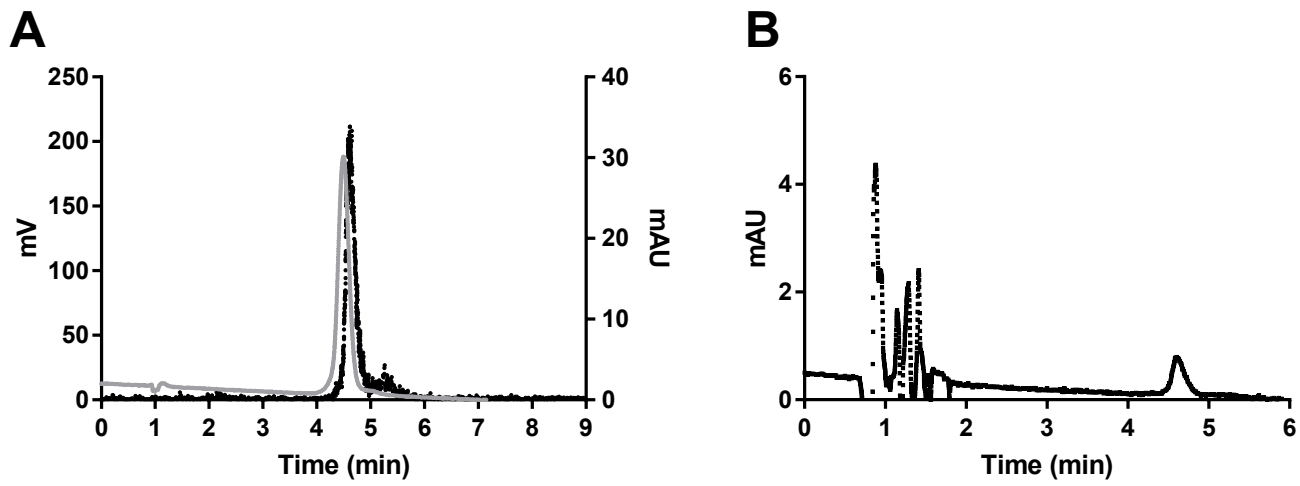

DS2OMe $\cdot\left[{ }^{11} \mathrm{C}\right] \mathrm{DS} 2 \mathrm{OMe}$

Supporting Figure S2: A) DS2OMe (1) (grey line) and $\left[{ }^{11} \mathrm{C}\right] \mathrm{DS} 2 \mathrm{OMe}\left(\left[{ }^{11} \mathrm{C}\right] \mathbf{1}\right)$ (black line) HPLC chromatograms. B) Product UV HPLC chromatogram

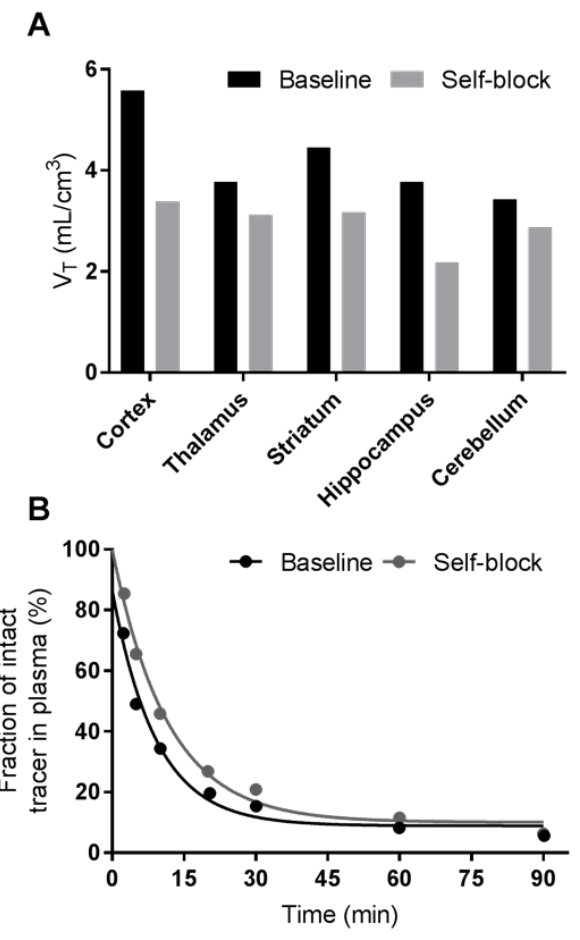

Supporting figure S3: A) Total distribution volumes $\left(\mathrm{V}_{T}\right)$ for the five indicated regions of interest at baseline (black) and after administration of of $2.1 \mathrm{mg} / \mathrm{kg}$ unlabeled $\mathbf{1}$ (co-administrated with the tracer). B) HPLC analysis of $\left.{ }^{11} \mathrm{C}\right] \mathbf{1}$ in pig plasma as a function of time after intravenous injection of $\left[{ }^{11} \mathrm{C}\right] \mathbf{1}$ (baseline, black symbols) or after administration of $2.1 \mathrm{mg} / \mathrm{kg}$ unlabeled $\mathbf{1}$ (co-administrated with the tracer, self-block, grey symbols). 


\section{Radiometabolism in pigs}

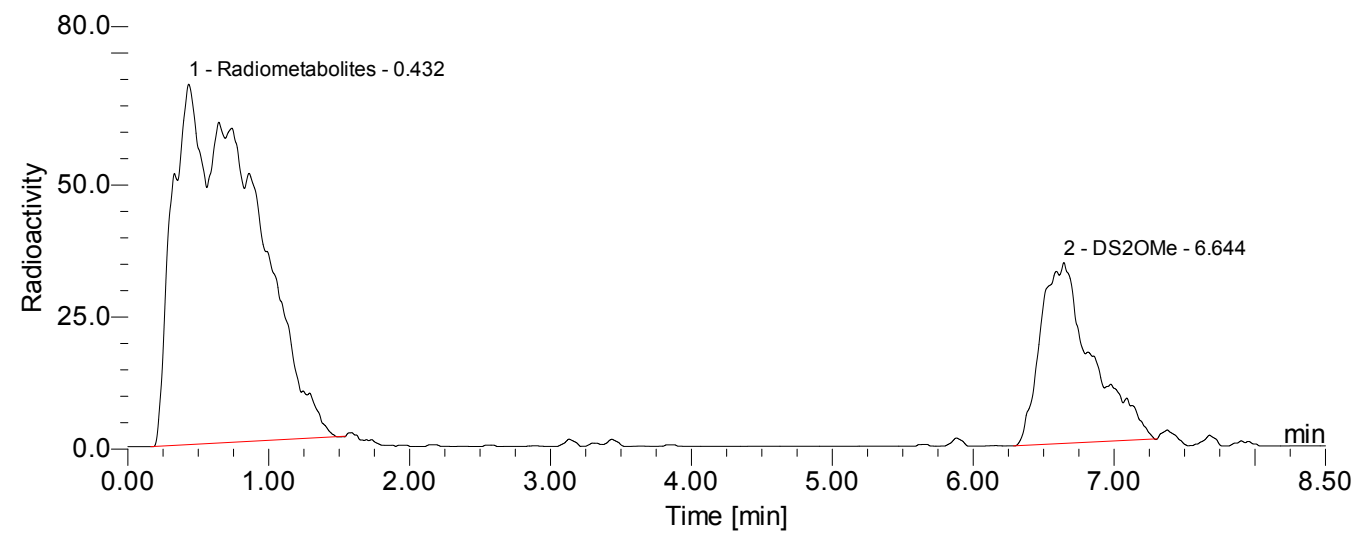

Supporting figure S4: Representative radio-chromatogram of pig plasma sample taken 20 min after injection with $\left[{ }^{11} \mathrm{C}\right] \mathrm{DS} 2 \mathrm{OMe}$. 